\title{
Do Statins Improve Survival in Small-Cell Lung Cancer?
}

\author{
Sanjay Popat, Royal Marsden Hospital and Imperial College London, London, United Kingdom
}

See accompanying article on page 1506

In an age when multiple therapeutic options are available, the cost of development and successful license is high, and with value in cancer care a key priority, ${ }^{1,2}$ the lure of repurposing established drugs is apparent, especially in cancer medicine. While Moore's law for microprocessors has demonstrated yearon-year ongoing efficiency, the reverse seems to be the case for new drugs, termed Eroom's law ${ }^{3}$ (Moore's law reversed), where the number of new drugs brought to market per billion US dollars spent on research and development has declined steadily. ${ }^{4}$ Therefore, the potential to repurpose therapeutic compounds for alternative uses is great and is a firmly established strategy in commercial development-an example being sildenafil, which was developed initially for angina and subsequently licensed for erectile dysfunction. ${ }^{5}$ However, a more attractive strategy is to redevelop an established licensed generic drug. In this circumstance, the safety profile is established, patents may have expired, which renders drugs cheap and available for trials, and new formulations or applications may result in additional commercial protection for new developers. Of importance, for oncology, the scope to improve cancer survival is wide.

It is against this backdrop that many provocative observational studies have investigated potential nonprimary benefits for 3-hydroxy-3-methylglutaryl coenzyme A inhibitors-statins-in cancer mortality or occurrence. Here, interest is high and multiple retrospective epidemiologic studies have suggested survival benefits for breast, colorectal, prostate, non-small-cell lung (nonSCLC), and pancreatic cancers. Whereas causality has not been established, a biologic rationale is plausible. Cholesterol is an important component of cell membranes and an integral component of lipid rafts, required for signaling complexes, cancer growth, and survival. It and related components of the mevalonate pathway, such as isopentenyl-diphosphate, farnesyl-diphosphate, geranylgeranyl-diphosphate, dolichol, and coenzyme Q, represent important metabolic modulators and are becoming increasingly recognized as potential therapeutic targets. ${ }^{6}$ Statins have been shown to be potentially therapeutic preclinically for cancerinhibiting tumor growth and inducing apoptosis in pancreatic, breast, mesothelioma, and SCLC cell lines-and may potentiate the efficacy of cytotoxic chemotherapy, either synergistically or additively. ${ }^{7,8}$ Specifically for SCLC, simvastatin demonstrated single-agent activity against $\mathrm{H}-69$ xenografts $^{7}$ as well as in conjunction with carboplatin or cisplatin.
In the article that accompanies this editorial, Seckl et $\mathrm{al}^{9}$ report the results of the LUNGSTAR trial, which was designed to test the hypothesis that statin use improves overall survival (OS) in patients with SCLC. In this study, 846 patients with either limited or extensive stage and performance status 0 to 3 were prospectively randomly assigned during a 5-year period to pravastatin $(40 \mathrm{mg}$ daily) or placebo for 2 years in a blinded manner, aiming to detect a hazard ratio (HR) of 0.82 assuming a median survival of 12 months. Patients were otherwise treated with standard platinum-etoposide chemotherapy with radiotherapy administered as per local practice. Disappointingly, and as is so often seen for SCLC, the results were unequivocally negative-OS was not improved (HR, 1.01; 95\% CI, 0.88 to $1.16 ; P=.90$ ), nor was progression-free survival (HR, 0.98 ; $95 \% \mathrm{CI}, 0.85$ to 1.13 ; $P=.81$ ). Lack of OS benefit did not differ between limited and extensive stage (interaction $P=.53$ ), and no differences in safety were noted. Whereas this important negative result merits widespread dissemination, several questions arise: did the control arm perform as expected? Was pravastatin the appropriate statin to use? Was the dosing of pravastatin optimal? Did primary care off-protocol statin use contribute to outcomes? What does this mean for patient care?

The LUNGSTAR investigators show that median OS for controls (10.6 months) underperformed trial expectations and, as would be expected, outperformed SCLC registry data ${ }^{10}$ and a previous trial that investigated thalidomide. ${ }^{11}$ Grade 3 to 5 toxicities were balanced between arms. During the period of recruitment, however, United Kingdom primary care was incentivized to optimize cardiovascular care in at-risk populations, including statin usage. Whereas the impact of this potential bias was not determined, it is likely minimal, as investigators specifically requested primary care not to prescribe statins during trial therapy, although specific data on this were not captured. Overall, given these data, major bias between arms is unlikely. The choice of pravastatin as intervention may have impacted the findings. Pravastatin is hydrophilic, unlike atorvastatin, simvastatin, and lovastatin-lipophilic compounds on which much of the retrospective observational cancer mortality data are based. In mice, atorvastatin, simvastatin, and lovastatin have been detected in extrahepatic tissues, including the brain, in both active acidic and inactive lactone forms in contrast to pravastatin, which was only detected in the liver, ${ }^{12}$ potentially suggesting a limited role for 
hydrophilic statins as anticancer agents. Moreover, other data sets have demonstrated a lack of benefit for pravastatin for cancer prevention or death. ${ }^{13}$ Was pravastatin dosing ideal? The licensed dosing for cardiovascular indications is 40 to $80 \mathrm{mg}$ per day, and the choice of $40 \mathrm{mg}$ used in LUNGSTAR is reasonable, as per previous cardiovascular studies that have retrospectively demonstrated survival benefits, although the maximum dose of $80 \mathrm{mg}$ may have been preferential.

Given the preclinical and epidemiologic data, why was no benefit with statin usage observed? Notwithstanding the potential biases above, several prospective, blinded trials have now also confirmed no benefit for statins in improving OS or progressionfree survival, as highlighted by the investigators themselves. ${ }^{9}$ LUNGSTAR is by far the largest prospective study to answer this question in any given cancer type and has definitely demonstrated no benefit. Was this because pravastatin did not have the desired biologic effect in LUNGSTAR? Unfortunately, no translational or corroborative studies were performed to demonstrate lowering of lipid levels and, indeed, whether abrogation of RAS superfamily function was observed; however, perhaps it is more likely that, given the pleiotropic biologic effects observed with statins in cancer, in vivo functional signaling redundancy rescues any potential anticancer effect observed in model systems.

In view of the important results from LUNGSTAR, where does this leave oncologists and our patients with SCLC? There is now clear evidence that adding pravastatin is of no benefit to standard therapy in treating patients with SCLC. Although not definitive, one may broaden this to other statins; however, as no obvious additional harm was identified, patients undergoing SCLC treatment who currently receive statins for cardiovascular indications should not be told to discontinue, although they would have been excluded from trial enrollment.

What does the future hold for statins in SCLC and repurposing in cancer? On the basis of these and other prospective trial data and given the resources required, additional prospective trials of statins to improve survival in other cancers are likely not justified. These data also fire another loud warning shot for cancer therapy repurposing, given the negative outcomes for thalidomide, ${ }^{11}$ topical nitroglycerin, ${ }^{14}$ and dalteparin. ${ }^{15}$ Moreover, for SCLC, where multiple trials have previously failed to deliver new systemic therapies despite encouraging retrospective or preclinical evidence, ${ }^{16}$ the systemic therapeutics future now eagerly anticipates late-phase development of immune-checkpoint inhibitors ${ }^{17}$ and antibody drug conjugates, ${ }^{18}$ which have both demonstrated early activity and have scope to markedly change the face of this resolutely stubborn disease.
AUTHOR'S DISCLOSURES OF POTENTIAL CONFLICTS OF INTEREST

Disclosures provided by the authors are available with this article at jco.org.

\section{REFERENCES}

1. Schnipper LE, Davidson NE, Wollins DS, et al: American Society of Clinical Oncology statement: A conceptual framework to assess the value of cancer treatment options. J Clin Oncol 33:2563-2577, 2015

2. Cherny NI, Sullivan R, Dafni $U$, et al: A standardised, generic, validated approach to stratify the magnitude of clinical benefit that can be anticipated from anti-cancer therapies: The European Society for Medical Oncology Magnitude of Clinical Benefit Scale (ESMO-MCBS). Ann Oncol 26:1547-1573, 2015

3. Scannell JW, Blanckley A, Boldon $H$, et al: Diagnosing the decline in pharmaceutical R\&D efficiency. Nat Rev Drug Discov 11:191-200, 2012

4. Munos B: Lessons from 60 years of pharmaceutical innovation. Nat Rev Drug Discov 8:959-968, 2009

5. Ghofrani HA, Osterloh $\mathrm{H}$, Grimminger F: Sildenafil: From angina to erectile dysfunction to pulmonary hypertension and beyond. Nat Rev Drug Discov 5: 689-702, 2006

6. Mullen PJ, Yu R, Longo J, et al: The interplay between cell signalling and the mevalonate pathway in cancer. Nat Rev Cancer 16:718-731, 2016

7. Khanzada UK, Pardo OE, Meier C, et al: Potent inhibition of small-cell lung cancer cell growth by simvastatin reveals selective functions of Ras isoforms in growth factor signalling. Oncogene 25:877-887, 2006

8. Chen J, Lan T, Hou J, et al: Atorvastatin sensitizes human non-small cell lung carcinomas to carboplatin via suppression of AKT activation and upregulation of TIMP-1. Int J Biochem Cell Biol 44:759-769, 2012

9. Seckl MJ, Ottensmeier $\mathrm{CH}$, Cullen $\mathrm{M}$, et al: Multicenter, phase III, randomized, double-blind, placebo-controlled trial of pravastatin added to first-line standard chemotherapy in small-cell lung cancer (LUNGSTAR). J Clin Oncol 35: 1506-1514, 2017

10. Lüchtenborg M, Riaz SP, Lim E, et al: Survival of patients with small cell lung cancer undergoing lung resection in England, 1998-2009. Thorax 69:269-273, 2014

11. Lee $S M$, Rudd R, Woll PJ, et al: Randomized double-blind placebo-controlled trial of thalidomide in combination with gemcitabine and carboplatin in advanced non-small-cell lung cancer. J Clin Oncol 27:5248-5254, 2009

12. Chen $C$, Lin J, Smolarek T, et al: P-glycoprotein has differential effects on the disposition of statin acid and lactone forms in mdr1a/b knockout and wild-type mice. Drug Metab Dispos 35:1725-1729, 2007

13. Hague WE, Simes J, Kirby A, et al: Long-term effectiveness and safety of pravastatin in patients with coronary heart disease: Sixteen years of follow-up of the LIPID study. Circulation 133:1851-1860, 2016

14. Davidson A, Veillard A-S, Tognela A, et al: A phase III randomized trial of adding topical nitroglycerin to first-line chemotherapy for advanced nonsmall-cell lung cancer: The Australasian lung cancer trials group NITRO trial. Ann Oncol 26: 2280-2286, 2015

15. Macbeth F, Noble S, Evans J, et al: Randomized phase III trial of standard therapy plus low molecular weight heparin in patients with lung cancer: FRAGMATIC trial. J Clin Oncol 34:488-494, 2016

16. Sharp A, Bhosle J, Abdelraouf F, et al: Development of molecularly targeted agents and immunotherapies in small cell lung cancer. Eur J Cancer 60:26-39, 2016

17. Antonia SJ, López-Martin JA, Bendell J, et al: Nivolumab alone and nivolumab plus ipilimumab in recurrent small-cell lung cancer (CheckMate 032): A multicentre, open-label, phase 1/2 trial. Lancet Oncol 17:883-895, 2016

18. Rudin CM, Pietanza MC, Bauer TM, et al: Rovalpituzumab tesirine, a DLL3targeted antibody-drug conjugate, in recurrent small-cell lung cancer: A first-inhuman, first-in-class, open-label, phase 1 study. Lancet Oncol 18:42-51, 2017

DOI: 10.1200/JCO.2016.72.0870; published at jco.org on March 6, 2017. 


\section{AUTHOR'S DISCLOSURES OF POTENTIAL CONFLICTS OF INTEREST}

\section{Do Statins Improve Survival in Small-Cell Lung Cancer?}

The following represents disclosure information provided by authors of this manuscript. All relationships are considered compensated. Relationships are self-held unless noted. I = Immediate Family Member, Inst = My Institution. Relationships may not relate to the subject matter of this manuscript. For more information about ASCO's conflict of interest policy, please refer to www.asco.org/rwc or ascopubs.org/jco/site/ifc.

\section{Sanjay Popat}

Honoraria: Merck, Pfizer

Consulting or Advisory Role: Boehringer Ingelheim, Eli Lilly, Novartis, Roche, Pfizer (Inst), Boehringer Ingelheim (Inst), Bristol-Myers

Squibb (Inst), Merck Sharp \& Dohme (Inst)

Research Funding: Boehringer-Ingelheim (Inst), Roche (Inst),

Bristol-Myers Squibb (Inst), Clovis Oncology (Inst)

Travel, Accommodations, Expenses: Boehringer Ingelheim, Merck Sharp

\& Dohme, Bristol-Myers Squibb 